\title{
Defining the Role of the Monoamine Oxidase-B Inhibitors for Parkinson's Disease
}

\author{
Daphne Robakis $^{1} \cdot$ Stanley Fahn ${ }^{1,2}$
}

Published online: 12 July 2015

(c) Springer International Publishing Switzerland 2015

\begin{abstract}
Inhibitors of monoamine oxidase-B (MAO-B) occupy an important place in the treatment of Parkinson's disease. Selegiline was the first MAO-B to be used therapeutically, while rasagiline is a second-generation drug with higher potency and selectivity. Safinamide is an investigational MAO-B inhibitor with non-dopaminergic properties that may provide advantages over its predecessors. As a class, MAO-B inhibitors are safe and well tolerated and provide symptomatic benefit both as monotherapy and in combination with other antiparkinsonian medications from early to late stages of disease. In combination with levodopa, MAO-B inhibitors may improve motor fluctuations and allow for lower total doses of levodopa. Patient characteristics and preferences can be important factors in deciding between agents. As a class, MAO-B inhibitors have shown promise as disease-modifying agents, but the clinical trial evidence to date has not been strong enough to afford them such a label. Future research may help further elucidate their relative merits and clarify their role in altering disease progression.
\end{abstract}

Stanley Fahn

sf1@columbia.edu

1 Department of Neurology, College of Physicians and Surgeons, Columbia University, New York, NY, USA

2 Neurological Institute, 710 West 168th Street, New York, NY 10032, USA

\section{Key Points}

Rasagiline and selegiline are two monoamine oxidase-B (MAO-B) inhibitors commonly used in the treatment of Parkinson's disease (PD), while safinamide is an investigational agent of the same class.

MAO-B inhibitors are safe, with few serious side effects, and provide mild but worthwhile improvements in the motor symptoms of PD, either as monotherapy or adjunctive to levodopa.

The possibility that MAO-B inhibitors could slow down the progression of $\mathrm{PD}$ has been suggested by some studies but not by others and remains an open question.

\section{Introduction}

Parkinson's disease (PD) is a common progressive neurological disorder currently affecting approximately $2 \%$ of the population over the age of 60 years [1]. Loss of dopaminergic cells in the substantia nigra results in striatal dopamine deficiency that produces a syndrome characterized by motoric disturbances, principally bradykinesia, rigidity, and rest tremor. Non-motor symptoms such as autonomic dysregulation, sleep problems, depression, anxiety, and cognitive changes can be debilitating and may occur in pre-symptomatic or late stages of disease. The most effective treatments for the motor symptoms of PD have been those that modulate the dopamine system, either 
by providing exogenous dopamine (levodopa), boosting dopaminergic activity (dopamine agonists), or interfering with dopamine catabolism (monoamine oxidase-B [MAOB] inhibitors). While there have been no revolutionary treatments for PD in the last few decades, great interest remains in the development of new drugs, novel delivery systems, and drug combinations, which are of utmost importance with the growing prevalence of the disease. MAO-B inhibitors are widely used in the management of both PD and psychiatric disorders and continue to be investigated for their therapeutic value and disease-modifying potential. This article discusses the characteristics and clinical use of the established (selegiline, rasagiline) and experimental (safinamide) MAO-B inhibitors in the treatment of PD.

\section{Monoamine Oxidase-B (MAO-B) Inhibitors}

Monoamine oxidase (MAO) is a mitochondrial enzyme that oxidatively deaminates monoamines (e.g., epinephrine, norepinephrine, serotonin, dopamine, tyramine, benzylamine, phenylethamine) and occurs in two isoforms, A and B [2]. Monoamine oxidase-A (MAO-A) shows greater activity towards deamination of serotonin and norepinephrine, while MAO-B shows greater activity towards benzylamine and phenylethylamine [3]. They are equally active towards dopamine and tyramine. Tyramine in the gut is deaminated by MAO-A, which is the main isoform found in non-central nervous system (CNS) organs. When MAO-A is inhibited, tyramine in the gut is not deaminated; after it is absorbed, it is taken up by the norepinephrine nerve terminals and acts as a false neurotransmitter. When it enters these nerve terminals, it displaces the stored norepinephrine. On its release, norepinephrine can create a hypertensive crisis. This crisis is known as the 'cheese effect' because it was initially found to be associated with the ingestion of certain cheeses. Subsequently, tyramine was identified as the specific ingredient that was the culprit. MAO-B does not act on tyramine in the gut, and therefore inhibitors of MAO-B may be used in the treatment of PD without the restriction of a low-tyramine diet. Both isoforms are present in the substantia nigra, with MAO-A being localized mainly to the pars compacta and MAO-B being the predominant isoform in the pars reticulata [4].

Dopamine is formed first by the conversion of dietary tyrosine to levodopa in neurons, which is then decarboxylated to dopamine in dopaminergic and noradrenergic neurons. When released from synaptic vesicles, dopamine is metabolized by MAO-B localized in the mitochondria glial cells surrounding the synaptic cleft. (In glia and postsynaptic neurons, dopamine is also metabolized by
catechol-O-methyl transferase [5]). MAO-B inhibitors thereby potentiate striatal neuronal responses to dopamine [6] and are therefore useful in the treatment of PD, whose underlying pathophysiology involves dopamine deficiency [7]. The two selective, irreversible MAO-B inhibitors currently approved by the US FDA for the treatment of PD are selegiline and rasagiline. Both selegiline and rasagiline are used in early PD as monotherapy and as adjunctive therapy to levodopa and dopamine agonists in later stages of PD $[8,9]$.

Compared with levodopa and dopamine agonists, the symptomatic relief of early PD provided by MAO-B inhibitors is modest at best [10]; however, they are associated with fewer side effects [11]. A study investigating monotherapy of rasagiline compared with ropinirole and pramipexole in early PD concluded that patients receiving rasagiline experienced fewer adverse events and dropout rates were lower than with those receiveing the dopamine agonists. Both ropinirole and pramipexole produced more adverse events related to gastrointestinal issues and sleep/fatigue, and pramipexole particularly was associated with a high incidence of adverse cognitive effects [12].

Levodopa is still the most effective medication for the treatment of PD. It is widely used [13] and, since its advent in 1967, has revolutionized the treatment of PD [14], improving the quality of life of millions of people worldwide [15]. However it is associated with motor complications such as wearing off, 'on-off' phenomena and dyskinesias after prolonged therapy for more than 5 years [16]. The possibility of levodopa toxicity or loss of efficacy over time has been entertained by many, and indeed some experimental models have demonstrated a toxic effect of levodopa on dopaminergic and serotonergic neurons in the brain $[17,18]$. Others have found that levodopa is not neurotoxic to animals, even at high doses [19], or to humans with PD [20], and may even promote neuronal recovery [21]. The ELLDOPA study, a multicenter placebo-controlled clinical trial, concluded that levodopa treatment did not accelerate the rate of PD progression and was possibly neuroprotective [22]. There is now compelling evidence that duration and severity of disease [23] and high dosages of levodopa [24] (but not duration of levodopa treatment) are major risk factors for inducing levodopa-induced dyskinesias. Although some investigators consider noncontinuous delivery of levodopa to be a risk factor for this complication, this has not been proven and is best considered a hypothesis. Because of concerns about developing dyskinesias with high-dosage levodopa, the desire to postpone the use of levodopa as long as possible is still a strategy favored by many patients and clinicians. 


\section{MAO-B Inhibitors and Neuroprotection}

One highly sought after goal for medical treatment of neurodegenerative disease, short of a cure, is the discovery of a drug that might retard disease pathology, rather than simply provide symptomatic relief. When first discovered, MAO-B inhibitors provided some hope of fulfilling the role of a disease-modifying agent in PD. The optimism was based largely on the fact that MAO-B metabolism produces oxygen free radicals, which supported the oxidative stress theory of neurodegeneration, and the observation that MAO-B helped attenuate damage from the neurotoxin $\mathrm{MPP}+. \quad \mathrm{N}$-Methyl-4-phenyl-1,2,3,6-tetrahydropyridine (MPTP) is a neurotoxin that is metabolized by MAO-B to the active form MPP+, which is toxic to dopaminergic neuron and causes PD-like symptoms in humans and animals. Selegiline, rasagiline, and safinamide have been shown to prevent MPTP from being oxidized to MPP+ $[25,26]$.

The mechanism of neuroprotection conferred by MAOB inhibitors has been hypothesized to be multifaceted based on in vitro studies, and may include prevention of reactive oxygen species production, increase of neurotrophic factors in neurons and glia, or upregulation of anti-apoptotic factors [27-29]. A related possibility is that MAO-B activity, which naturally increases with age [30], is itself dysfunctional and that excessive or even normal activity is a potential cause of oxidative stress in PD [31]. However, demonstrating disease modification in human studies is challenging as it requires a study design that differentiates between improvements that result from symptomatic benefit and those that result from altering the natural history of the disease. The FDA's requirement for proving disease modification has been quite rigorous. The higher dose of rasagiline met only one of three hierarchical endpoints required to prove disease modification in the ADAGIO study and was therefore ultimately rejected by the FDA as a disease-modifying agent [32]. Other drugs have similarly failed to achieve disease-modifying status when translated from the laboratory to human beings [33].

\section{Safety of MAO-B Inhibitors}

MAO-B inhibitors are quite safe and well tolerated as a class, with few drug-drug interactions. One potentially serious complication from taking high doses of MAO inhibitors is the hypertensive 'cheese effect', as mentioned above. This is a concern for non-selective MAO inhibitors that had been used to treat depression or for high doses of relatively selective MAO-B inhibitors to the extent that MAO-A is also inhibited, and serves as the basis for the recommendation that dietary tyramine be avoided by patients treated with MAO inhibitors. However, at the recommended doses of the MAO-B inhibitors used in the treatment of PD, they are highly selective for MAO-B and therefore obviate the need for dietary control [34]. Selegiline is dosed at $10 \mathrm{mg} /$ day for the treatment of PD, and does not produce MAO-A inhibition (and therefore, tyramine potentiation) until a dose of $30 \mathrm{mg} /$ day is reached [35-37]. Likewise, rasagiline at the approved doses of $0.5-1 \mathrm{mg} /$ day is not associated with any significant tyramine pressor response [38, 39]. Restrictions of tyramine consumption are no longer viewed as necessary [37, 40].

Serotonin syndrome is another serious adverse reaction that can result from loss of MAO-B selectivity during the concomitant administration of an MAO-B inhibitor and a serotonergic medication. This syndrome is potentially fatal and is caused by a toxic build-up of serotonin, leading to fever, hallucinations, tachycardia, and gastrointestinal symptoms. However, the high selectivity of MAO-B inhibitors makes this an exceptional occurrence. A survey of the Parkinson Study Group revealed that only $0.24 \%$ of patients treated with selegiline + a selective serotonin reuptake inhibitor (SSRI) developed symptoms possibly consistent with serotonin syndrome, and only $0.04 \%$ experienced a serious reaction, with no fatalities [41]. In the PRESTO study of rasagiline, no difference was found in the rate of adverse events between SSRI-treated and non-SSRI-treated patients [42]. Recently, a large retrospective cohort study found no cases of serotonin syndrome in patients treated with rasagiline plus an antidepressant [41]. Therefore, in most cases, MAO-B inhibitors can be safely administered along with SSRIs. This is important because the co-morbidity of depression and PD makes it quite likely that an SSRI will be required at some point during the disease course [43-45]. Discontinuing a MAO-B inhibitor in order to start an SSRI or using an alternative class of antidepressant to avoid interaction is generally not necessary as long as the recommended dose of MAO-B inhibitor is not exceeded.

\subsection{Selegiline}

Knoll and Magyar described selegiline in 1965 and showed it was a selective MAO-B inhibitor and did not cause the cheese effect [46]. Selegiline was the first MAO$\mathrm{B}$ inhibitor to be approved by the FDA for the treatment of $\mathrm{PD}$, and it is used as monotherapy and as an adjunct in moderately advanced PD. It is a selective, irreversible MAO-B inhibitor and, unlike rasagiline, is metabolized to $\mathrm{L}$-methamphetamine and $\mathrm{L}$-amphetamine.

Clinical studies and experimental and animal models in the 1980s suggested that selegiline may delay disease progression [47], perhaps by reducing oxidative stress-related pathways causing dopaminergic neuronal death in the 
substantia nigra [48]. This led to the prospective investigation of the effect of selegiline on the natural progression of PD [26], and to the extensive DATATOP study, the first double-blind placebo-controlled neuroprotection trial [49].

DATATOP enrolled 800 patients with newly diagnosed PD and randomized them to receive either deprenyl (an earlier name for selegiline) (10 mg), tocopherol (2000 units), or placebo. After 12 months, $47 \%$ of those treated with placebo needed to start levodopa compared with $26 \%$ in the selegiline group. Selegiline delayed the need for symptomatic treatment with levodopa by 9 months. Due to a mild symptomatic benefit from selegiline (improvement of about 3 points on the total Unified Parkinson's Disease Rating Scale [UPDRS] compared with placebo after 3 months), doubt remained whether these results could be used to support a disease-modifying effect. Other trials have had similar results $[50,51]$.

A long-term follow-up study to DATATOP re-randomized 368 patients who had already come to require Ldopa to either placebo or selegiline for 2 years [52]. The selegiline group experienced slower progression of motor symptoms by UPDRS score and also had less freezing of gait (FOG) $(16 \%)$ than the placebo group (29\%), although the rate of dyskinesias was higher (34 vs. $19 \%$ ).

Several long-term clinical trials have found that the combination of selegiline plus levodopa is associated with decreased levodopa requirements over time than levodopa alone [53-55]. The prospective double-blind SINDEPAR study attempted to resolve the question of neuroprotection by utilizing a washout period for selegiline [56]. It compared initially untreated PD patients taking selegiline plus levodopa against those receiving selegiline plus placebo and measured their UPDRS scores after 2 months of no selegiline and 7 days of no levodopa (washout). The selegiline group deteriorated significantly less in their total UPDRS scores compared with placebo even after they were off selegiline for 2 months. Still, many found the washout period to be insufficiently long to provide conclusive evidence of neuroprotection, as the benefit could be explained by a long-term symptomatic benefit [57]. The washout period required to eliminate a symptomatic effect is still controversial; the half-life of selegiline has been found to be anywhere from 2 to 40 days, depending on the study [58]. In addition to lowering levodopa requirements, selegiline has been associated with slower disease progression as measured by Hoehn and Yahr stage [59] and with slower accumulation of disability [54].

Selegiline is dosed at $10 \mathrm{mg}$ per day divided into twicedaily doses. In some patients, the amphetamine metabolites may interfere with sleep, as insomnia is one of the most common side effects of selegiline [60], although this can usually be prevented by avoiding evening dosing. The stimulant properties of selegiline can sometimes be used to therapeutic effect in patients experiencing daytime fatigue, an extremely common complaint in PD [61, 62]. In summary, selegiline has mild symptomatic benefits for PD and good evidence for producing delay in disease progression and reduction in requirement for levodopa.

\subsection{Rasagiline}

Rasagiline is a selective irreversible MAO-B inhibitor approved by the FDA in 2006 for the treatment of PD. It is dosed once daily, and can be used alone or in combination with other PD medications from early to late stages of the disease. Rasagiline is highly selective for MAO-B versus MAO-A at the doses used for the treatment of PD (0.5-1 mg/day), more so than selegiline, and does not possess any other significant type of pharmacologic effects [63]. The therapeutic efficacy of rasagiline has been demonstrated in a number of phase III-IV randomized double-blind placebo-controlled multicenter trials [32, 42, $64,65]$. As adjunctive therapy, rasagiline has a significant beneficial effect on motor symptoms compared with placebo as measured by UPDRS scores and significantly decreases daily 'off' time [42, 65]. The TEMPO and ADAGIO trials also addressed the potential disease-modifying effect of rasagiline by employing the delayed-start design.

The TEMPO trial examined rasagiline efficacy as initial therapy in 404 patients with early PD not yet requiring dopaminergic therapy [66]. Patients were randomized to receive treatment with either rasagiline 1 or $2 \mathrm{mg}$ or placebo for 26 weeks. After 26 weeks, the placebo group was re-randomized to receive rasagiline $2 \mathrm{mg}$ for another 26 weeks (delayed-start group), while the initial rasagiline group continued with either 1 or $2 \mathrm{mg}$. The primary outcome measure for the first phase of the study was change in total UPDRS motor score for the treatment versus the placebo group before and after treatment. The mean difference between the early and delayed start groups was about 2 units on the UPDRS in favor of early start after 1 year [66]. Over a 6.5-year open-label follow-up period, the difference persisted at 2.5 units [67]. The ADAGIO study was designed to more rigorously address the question of disease modification with stricter outcome measures, a larger study group (1176 patients), and a longer delayedstart period. Evidence was seen of a mild neuroprotective effect with rasagiline $1 \mathrm{mg}$ but not $2 \mathrm{mg}$ [31]. However, a post hoc analysis revealed that both doses significantly delayed the need for additional dopaminergic therapy. It is unclear how long these effects persist since, after 72 weeks, the only difference between the delayed and early-start groups was in the Activities of Daily Living (ADL) subscore [68]. Rasagiline has been conclusively demonstrated to improve motor function in PD over at least 
5 years, but any potential neuroprotective benefit has yet to be confirmed.

\subsection{Safinamide}

Safinamide is another MAO-B inhibitor recently approved by the European Commission as an add-on to levodopa or in combination with other PD medications in mid- to latestage PD patients with motor fluctuations. It is currently under review by the FDA for use in both early and mid- to late-stage PD at doses of 50 and $100 \mathrm{mg}$ per day. Safinamide is so selective for MAO-B that even doses of $10 \mathrm{mg} / \mathrm{kg}$ do not produce inhibition of MAO-A [69]. Similar to rasagiline and selegiline, it is an irreversible inhibitor; however, unlike them, it also has additional pharmacologic properties. It blocks voltage-dependent sodium channels and inhibits glutamate release [70].

The efficacy of safinamide has been established for early and advanced PD in phase II and phase III trials. Two phase III clinical trials have been conducted in early PD with patients receiving dopamine agonists and two in midto late-stage $\mathrm{PD}$ for patients receiving levodopa with motor fluctuations [71-74]. In a 24-week randomized placebocontrolled trial of patients with early PD, the $100 \mathrm{mg} / \mathrm{day}$ dose of safinamide was associated with significant improvement in UPDRS part III and part II (ADL scale), and the Clinical Global Impression scale compared with placebo, while the $200 \mathrm{mg} /$ day was not [71]. The MOTION trial evaluated safinamide 50 and $100 \mathrm{mg}$ /day as an add-on to a dopamine agonist in early $\mathrm{PD}$ and demonstrated improvement in UPDRS III and quality of life in the PD questionnaire (PDQ-39) measures compared with placebo for the 100-mg dose [72]. Finally, the SETTLE trial was a double-blind placebo-controlled randomized controlled trial that found safinamide was effective in improving ADL scores and emotional well-being in patients with mid- to late-stage idiopathic PD and motor fluctuations on stable levodopa therapy [73], thus supporting results found in study 016 [74].

A potential advantage of safinamide over the other MAO-B inhibitors is a purported antidyskinetic effect, which is thought to be related to its anti-glutamate activity [25]. The antidyskinetic properties have been demonstrated in an animal model on MTPP-lesioned dyskinetic macaque monkeys, where pretreatment with safinamide both reduced dyskinesias and prolonged the duration of the antiparkinsonian effect of levodopa. Amantadine alone was effective in reducing dyskinesias but also reduced the motor effect of levodopa [75]. Amantadine is currently the only commonly prescribed antidyskinetic medication, and it is not effective in all patients with dyskinesias [76]. In the extension study of the phase III trial for mid- to latestage PD (study 016), safinamide did not significantly improve scores on the dyskinesia rating scale compared with placebo after 2 years, but scores did improve in a subgroup of patients receiving safinamide $100 \mathrm{mg}$ with moderate to severe dyskinesias. More work is needed to elucidate the potential antidyskinetic properties of safinamide in PD patients [77].

As with the other MAO-B inhibitors, safinamide has neuroprotective properties in vitro and in vivo. It has been shown to prevent neuronal cell death in animal and tissue culture models [25]. Its antiglutamate effect may provide an additional route of neuroprotective benefit, as glutamate is known to be toxic to neurons [78]. At high doses, safinamide has been shown to protect against hippocampal neuron loss when given to rodents 15 min before kainic acid, a glutamate analog [25]. In a study on rats with experimental autoimmune encephalitis, safinamide protected against neurological disability and axonal degeneration [79].

The ion channel blockade and consequent diminution in glutamate release caused by safinamide occur at brain concentrations higher than those required for MAO-B inhibition, which raises the question of whether safinamide's therapeutic efficacy is attributable solely to MAO-B inhibition. Clinical studies that have increased the dose of safinamide beyond that calculated to provide complete MAO-B inhibition $(\geq 0.3 \mathrm{mg} / \mathrm{kg})$ have shown that these higher doses provide further clinical benefit and that additional dose increases correspond with more benefit [80, 81]. Therefore both laboratory and clinical evidence exists that safinamide is mechanistically different than other MAO-B inhibitors. This could provide significant advantages over selegiline and rasagiline, especially if safinamide's potential as a neuroprotective and anti-dyskinetic agent are realized. Another next-generation MAO-B inhibitor, HT-3951 is currently in development by Dart Neuroscience and is in phase I studies.

\section{Combination Therapies}

MAO-B-inhibitors are frequently used in combination with other dopaminergic and non-dopaminergic medications from early- through late-stage PD. While their symptomatic benefit becomes less noticeable as the disease progresses, they may be useful in prolonging the effect of levodopa, in reducing the total dose of levodopa, and in improving 'on-off' motor fluctuations. Selegiline plus levodopa has been found to be superior to levodopa monotherapy [55], and the LARGO and PRESTO trials showed that rasagiline was better than placebo in decreasing 'off' time [42, 65]. Additionally, LARGO showed that the effects of rasagiline were equivalent to those of entacapone in reducing 'off' time. Recently, 
rasagiline was shown to be an effective adjunct to dopamine agonists (ropinirole and pramipexole) in improving motor symptoms [82]. Likewise, evidence is now growing that safinamide can be effectively added to both dopamine agonists and levodopa in mid- to late-stage PD [71, 73, 74, $77,83]$. MAO-B inhibitors can be successfully combined with almost any class of antiparkinson medications due to their low risk for drug-drug interactions, and, as adjunct therapy, they constitute a valuable addition to a clinician's drug armamentarium.

\section{Treatment Decisions: Rasagiline Versus Selegiline}

No head-to-head randomized clinical trials have compared the efficacy of rasagiline with that of selegiline in the treatment of PD, but several studies have attempted to answer this question through indirect comparative methods. A meta-analysis by Stowe et al. [10] found a significantly greater reduction in L-dopa dose with selegiline than with rasagiline but noted that the levodopa dose in the rasagiline trials could be adjusted only within the first 6 weeks of the trial, while adjustments were permitted throughout the entire study in the selegiline trial, thus concluding that evidence was insufficient to posit any differences between the two drugs [10]. More recently, Marconi and Zwingers [84] compared the efficacy of selegiline and rasagiline in the treatment of early PD by comparing the pooled results of nine clinical trials using forest plot analysis and standardized mean differences of change in total UPDRS score [84]. They found that both drugs were more effective than placebo but were not statistically different from each other in reducing total UPDRS scores from baseline up to 9 months after treatment initiation. On the other hand, Jost et al. [85] conducted a similar retrospective comparative meta-analysis with less selective criteria for study inclusion and reported a distinct advantage of rasagiline over selegiline in terms of total UPDRS score. They also reported that the study discontinuation rate due to adverse side effects was significantly higher with selegiline than with rasagiline [86].

Both selegiline and rasagiline have been examined for their effect on FOG, a particularly intractable and debilitating symptom of advanced PD [87]. In the controlled BLIND-DATE trial, an extension study of DATATOP, Shoulson et al. [52] found that fewer patients treated with selegiline developed FOG than those treated with placebo, which was thought to be unrelated to a symptomatic dopaminergic effect since patients in both treatment arms were already being treated with levodopa and could receive as much levodopa as the treating investigator considered necessary . In the LARGO study, rasagiline was found to significantly improve UPDRS FOG subscores in patients who already had FOG at the start of the study [65]. However, the effects are quite small ( -0.16 UPDRS points in the case of the LARGO study) and, even if reproducible, are of unclear clinical significance. Still, given the absence of any proven treatment for freezing, any hint of a clinical benefit, either symptomatic or neuroprotective, may be worth considering by clinicians.

In terms of efficacy as measured by improvement of motor symptoms or FOG, it is therefore difficult to argue in favor of the superiority of either rasagiline or selegiline for the purposes of clinical practice. From a disease-modification standpoint, the delayed-start study design used in the rasagiline ADAGIO trial was better at teasing apart a disease-modifying effect from a symptomatic effect than was the DATATOP selegiline trial design, but still could not convincingly demonstrate a disease-modifying effect [88]. While ADAGIO did demonstrate a reduced cumulative disability in the early- versus late-start group over 18 months, why this benefit was seen with the 1-mg/day and not the 2-mg/day test dose remains unclear and casts doubt on the significance of the improvement associated with the lower dose. In addition, the duration of benefit of the rasagiline $1-\mathrm{mg} /$ day dose remains speculative beyond the 18-month period that has been formally studied. Nevertheless, these data may be used to support the practice of early treatment with an MAO-B inhibitor to delay motor deterioration via alternative mechanisms such as the postponement of deleterious physiologic changes in the brain and basal ganglia circuitry resulting from chronic dopamine deficiency [89]. Supplying dopamine to neurons may support the intrinsic compensatory mechanisms that are at work, for example, for a period of time in early PD at which point $80 \%$ of striatal dopamine has already been lost $[89,90]$.

The problem with this hypothesis is that pramipexole failed to be different from placebo in a delayed-start study [33]. Therefore, the compensatory mechanism as being neuroprotective is doubtful.

Based on the clinical data available to date, it is difficult to find a clear therapeutic or neuroprotective advantage, or clinically relevant safety advantage for either rasagiline or selegiline, although the debate on this topic continues [91, 92]. Patient characteristics and preferences or practical issues may therefore come into play when choosing an agent, for example, cost, desire to either avoid or harness amphetamine metabolites, desire for a 'newer' drug versus one with a longer prescribing history, the experience of the prescribing physician with the medication, or dosing frequency [93]. 


\section{Conclusions}

MAO-B inhibitors were introduced for the treatment of Parkinson's disease over 40 years ago and remain a popular mainstay of treatment. While they do not provide the immediate, powerful antiparkinsonian effect of levodopa, they offer mild symptomatic improvement without the complications associated with levodopa and therefore may be particularly useful in the treatment of early or mild PD. In advanced PD, they can be beneficial in ameliorating 'onoff' motor fluctuations and limiting levodopa requirements. Further research is needed directly comparing the efficacy and side effect profiles of the different MAO-B inhibitors before clinicians can make informed recommendations regarding agent selection. The pre- and post-clinical evidence in favor of a neuroprotective and disease-modifying effect for MAO-B inhibitors is in no way conclusive, but it is nevertheless unparalleled by any other class of antiparkinsonian medications to date. The possibility that MAO-B inhibitors could slow the progression of PD provides additional incentive to start treatment with these agents early in the disease course and to continue in the long term. The promise of neuroprotection has continued to spawn interest in the development and testing of new drugs with MAO-B inhibitory as well as non-dopaminergic activity. As we advance our understanding of neurodegenerative diseases and the complex pathophysiology of PD, the basis for discovery of a definitive neuroprotective agent should come within closer reach.

\section{Compliance with Ethical Standards}

Funding No funding was specifically received for the conduct or publication of this review.

Conflict of interest The authors, Daphne Robakis and Stanley Fahn, declare that they have no conflicts of interest and no competing financial interests.

\section{References}

1. de Lau LM, Breteler MM. Epidemiology of Parkinson's disease. Lancet Neurol. 2006;5(6):525-35.

2. Johnston JP. Some observations upon a new inhibitor of monoamine oxidase in brain tissue. Biochem Pharmacol. 1968;17(7):1285-97.

3. Kalgutkar AS, Dalvie DK, Castagnoli N Jr, Taylor TJ. Interactions of nitrogen-containing xenobiotics with monoamine oxidase (MAO) isozymes A and B: SAR studies on MAO substrates and inhibitors. Chem Res Toxicol. 2001;14(9):1139-62.

4. Saura Marti J, Kettler R, Da Prada M, Richards JG. Molecular neuroanatomy of MAO-A and MAO-B. J Neural Transm Suppl. 1990;32:49-53.

5. Kaakkola S, Mannisto PT, Nissinen E. Striatal membrane-bound and soluble catechol- $O$-methyl-transferase after selective neuronal lesions in the rat. J Neural Transm. 1987;69(3-4):221-8.
6. Berry MD, Scarr E, Zhu MY, Paterson IA, Juorio AV. The effects of administration of monoamine oxidase- $\mathrm{B}$ inhibitors on rat striatal neurone responses to dopamine. $\mathrm{Br} \mathrm{J}$ Pharmacol. 1994;113(4):1159-66.

7. Fernandez HH, Chen JJ. Monoamine oxidase-B inhibition in the treatment of Parkinson's disease. Pharmacotherapy. 2007;27(12 Pt 2): 174 s-85s.

8. Selegiline Hydrochloride. Lexi-drugs online. Hudson, OH: LexiComp, Inc. http://online.lexi.com/lco/action/doc/retrieve/docid/ patch_f/7655?hl=6940. Accessed 25 Jan 2015.

9. US FDA. Azilect (rasagiline mesylate): US prescribing information. 2014. http://www.accessdata.fda.gov/drugsatfda_docs/ label/2014/021641s016s017lbl.pdf. Accessed 25 Jan 2015.

10. Stowe R, Ives N, Clarke CE, Handley K, Furmston A, Deane K, et al. Meta-analysis of the comparative efficacy and safety of adjuvant treatment to levodopa in later Parkinson's disease. Mov Disord. 2011;26(4):587-98.

11. Caslake R, Macleod A, Ives N, Stowe R, Counsell C. Monoamine oxidase B inhibitors versus other dopaminergic agents in early Parkinson's disease. Cochrane Database Syst Rev. 2009(4):Cd006661.

12. Zagmutt FJ, Tarrants ML. Indirect comparisons of adverse events and dropout rates in early Parkinson's disease trials of pramipexole, ropinirole, and rasagiline. Int $\mathrm{J}$ Neurosci. 2012;122(7):345-53.

13. Stacy M. Medical treatment of Parkinson disease. Neurol Clin. 2009;27(3):605-31, v.

14. Fahn S. The medical treatment of Parkinson disease from James Parkinson to George Cotzias. Mov Disord. 2015 Jan;30(1):4-18.

15. Hornykiewicz O. A brief history of levodopa. J Neurol. 2010;257(Suppl 2):S249-52.

16. Fabbrini G, Brotchie JM, Grandas F, Nomoto M, Goetz CG. Levodopa-induced dyskinesias. Mov Disord. 2007;22(10):1379-89 (quiz 523).

17. Asanuma M, Miyazaki I, Ogawa N. Dopamine- or L-DOPA-induced neurotoxicity: the role of dopamine quinone formation and tyrosinase in a model of Parkinson's disease. Neurotox Res. 2003;5(3):165-76.

18. Stansley BJ, Yamamoto BK. L-dopa-induced dopamine synthesis and oxidative stress in serotonergic cells. Neuropharmacology. 2013;67:243-51.

19. Perry TL, Yong VW, Ito M, Foulks JG, Wall RA, Godin DV, et al. Nigrostriatal dopaminergic neurons remain undamaged in rats given high doses of L-DOPA and carbidopa chronically. J Neurochem. 1984;43(4):990-3.

20. Parkkinen L, O’Sullivan SS, Kuoppamaki M, Collins C, Kallis C, Holton JL, et al. Does levodopa accelerate the pathologic process in Parkinson disease brain? Neurology. 2011;77(15):1420-6.

21. Murer MG, Dziewczapolski G, Menalled LB, Garcia MC, Agid $\mathrm{Y}$, Gershanik $\mathrm{O}$, et al. Chronic levodopa is not toxic for remaining dopamine neurons, but instead promotes their recovery, in rats with moderate nigrostriatal lesions. Ann Neurol. 1998;43(5):561-75.

22. Fahn S. Does levodopa slow or hasten the rate of progression of Parkinson's disease? J Neurol. 2005;252 Suppl 4:Iv37-42.

23. Cilia R, Akpalu A, Sarfo FS, Cham M, Amboni M, Cereda E, et al. The modern pre-levodopa era of Parkinson's disease: insights into motor complications from sub-Saharan Africa. Brain. 2014;137(Pt 10):2731-42.

24. Olanow CW, Kieburtz K, Rascol O, Poewe W, Schapira AH, Emre M, et al. Factors predictive of the development of Levodopa-induced dyskinesia and wearing-off in Parkinson's disease. Mov Disord. 2013;28(8):1064-71.

25. Caccia C, Maj R, Calabresi M, Maestroni S, Faravelli L, Curatolo L, et al. Safinamide: from molecular targets to a new antiParkinson drug. Neurology. 2006;67(7 Suppl 2):S18-23. 
26. Tetrud JW, Langston JW. The effect of deprenyl (selegiline) on the natural history of Parkinson's disease. Science (New York, NY). 1989;245(4917):519-22.

27. Boll MC, Alcaraz-Zubeldia M, Rios C. Medical management of Parkinson's disease: focus on neuroprotection. Curr Neuropharmacol. 2011;9(2):350-9.

28. Tatton W, Chalmers-Redman R, Tatton N. Neuroprotection by deprenyl and other propargylamines: glyceraldehyde-3-phosphate dehydrogenase rather than monoamine oxidase B. J Neural Transm. 2003;110(5):509-15.

29. Wu RM, Chen RC, Chiueh CC. Effect of MAO-B inhibitors on MPP+ toxicity in vivo. Ann N Y Acad Sci. 2000;899:255-61.

30. Kumar MJ, Andersen JK. Perspectives on MAO-B in aging and neurological disease: where do we go from here? Mol Neurobiol. 2004;30(1):77-89.

31. Kandadai RM, Jabeen SA, Kanikannan MA, Borgohain R. Safinamide for the treatment of Parkinson's disease. Expert Rev Clin Pharmacol. 2014;7(6):747-59.

32. Olanow CW, Rascol O, Hauser R, Feigin PD, Jankovic J, Lang A, et al. A double-blind, delayed-start trial of rasagiline in Parkinson's disease. N Engl J Med. 2009;361(13):1268-78.

33. Schapira AH, McDermott MP, Barone P, Comella CL, Albrecht $\mathrm{S}$, Hsu HH, et al. Pramipexole in patients with early Parkinson's disease (PROUD): a randomised delayed-start trial. Lancet Neurol. 2013;12(8):747-55.

34. Finberg JP. Update on the pharmacology of selective inhibitors of MAO-A and MAO-B: focus on modulation of CNS monoamine neurotransmitter release. Pharmacol Ther. 2014;143(2): $133-52$.

35. Finberg JP, Tenne M. Relationship between tyramine potentiation and selective inhibition of monoamine oxidase types $\mathrm{A}$ and $\mathrm{B}$ in the rat vas deferens. Br J Pharmacol. 1982;77(1):13-21.

36. Mann JJ, Aarons SF, Wilner PJ, Keilp JG, Sweeney JA, Pearlstein $\mathrm{T}$, et al. A controlled study of the antidepressant efficacy and side effects of (-)-deprenyl. A selective monoamine oxidase inhibitor. Arch Gen Psychiatry. 1989;46(1):45-50.

37. Chen JJ, Wilkinson JR. The monoamine oxidase type B inhibitor rasagiline in the treatment of Parkinson disease: is tyramine a challenge? J Clin Pharmacol. 2012;52(5):620-8.

38. deMarcaida JA, Schwid SR, White WB, Blindauer K, Fahn S, Kieburtz K, et al. Effects of tyramine administration in Parkinson's disease patients treated with selective MAO-B inhibitor rasagiline. Mov Disord. 2006;21(10):1716-21.

39. Goren T, Adar L, Sasson N, Weiss YM. Clinical pharmacology tyramine challenge study to determine the selectivity of the monoamine oxidase type B (MAO-B) inhibitor rasagiline. J Clin Pharmacol. 2010;50(12):1420-8.

40. McCormack PL. Rasagiline: a review of its use in the treatment of idiopathic Parkinson's disease. CNS Drugs. 2014;28(11): 1083-97.

41. Panisset M, Chen JJ, Rhyee SH, Conner J, Mathena J. Serotonin toxicity association with concomitant antidepressants and rasagiline treatment: retrospective study (STACCATO). Pharmacotherapy. 2014;34(12):1250-8.

42. Parkinson Study Group. A randomized placebo-controlled trial of rasagiline in levodopa-treated patients with Parkinson disease and motor fluctuations: the PRESTO study. Arch Neurol. 2005;62(2):241-8.

43. Reijnders JS, Ehrt U, Weber WE, Aarsland D, Leentjens AF. A systematic review of prevalence studies of depression in Parkinson's disease. Mov Disord. 2008;23(2):183-9 (quiz 313).

44. Richard IH, McDermott MP, Kurlan R, Lyness JM, Como PG, Pearson N, et al. A randomized, double-blind, placebo-controlled trial of antidepressants in Parkinson disease. Neurology. 2012;78(16):1229-36.
45. Weintraub D, Moberg PJ, Duda JE, Katz IR, Stern MB. Recognition and treatment of depression in Parkinson's disease. J Geriatr Psychiatry Neurol. 2003;16(3):178-83.

46. Knoll J, Magyar K. Some puzzling pharmacological effects of monoamine oxidase inhibitors. Adv Biochem Psychopharmacol. 1972;5:393-408.

47. Birkmayer W, Knoll J, Riederer P, Youdim MB, Hars V, Marton $\mathrm{J}$. Increased life expectancy resulting from addition of L-deprenyl to Madopar treatment in Parkinson's disease: a longterm study. J Neural Transm. 1985;64(2):113-27.

48. Gerlach M, Youdim MB, Riederer P. Pharmacology of selegiline. Neurology. 1996;47(6 Suppl 3):S137-45.

49. Parkinson Study Group. Effects of tocopherol and deprenyl on the progression of disability in early Parkinson's disease. N Engl J Med. 1993;328(3):176-83.

50. Allain H, Pollak P, Neukirch HC. Symptomatic effect of selegiline in de novo Parkinsonian patients. The French Selegiline Multicenter Trial. ement disorders. Mov Disord. 1993;8(Suppl 1):S36-40.

51. Myllyla VV, Sotaniemi KA, Vuorinen JA, Heinonen EH. Selegiline as initial treatment in de novo parkinsonian patients. Neurology. 1992;42(2):339-43.

52. Shoulson I, Oakes D, Fahn S, Lang A, Langston JW, LeWitt P, et al. Impact of sustained deprenyl (selegiline) in levodopatreated Parkinson's disease: a randomized placebo-controlled extension of the deprenyl and tocopherol antioxidative therapy of parkinsonism trial. Ann Neurol. 2002;51(5):604-12.

53. Larsen JP, Boas J, Erdal JE. Does selegiline modify the progression of early Parkinson's disease? Results from a five-year study. The Norwegian-Danish Study Group. Eur J Neurol. 1999;6(5):539-47.

54. Palhagen S, Heinonen E, Hagglund J, Kaugesaar T, Maki-Ikola O, Palm R. Selegiline slows the progression of the symptoms of Parkinson disease. Neurology. 2006;66(8):1200-6.

55. Przuntek H, Conrad B, Dichgans J, Kraus PH, Krauseneck P, Pergande G, et al. SELEDO: a 5-year long-term trial on the effect of selegiline in early Parkinsonian patients treated with levodopa. Eur J Neurol. 1999;6(2):141-50.

56. Olanow CW, Hauser RA, Gauger L, Malapira T, Koller W, Hubble $\mathrm{J}$, et al. The effect of deprenyl and levodopa on the progression of Parkinson's disease. Ann Neurol. 1995;38(5):771-7.

57. Stocchi F, Olanow CW. Neuroprotection in Parkinson's disease: clinical trials. Ann Neurol. 2003;53 Suppl 3:S87-97 (discussion S-9).

58. Riederer P, Lachenmayer L. Selegiline's neuroprotective capacity revisited. J Neural Transm. 2003;110(11):1273-8.

59. Zhao YJ, Wee HL, Au WL, Seah SH, Luo N, Li SC, et al. Selegiline use is associated with a slower progression in early Parkinson's disease as evaluated by Hoehn and Yahr stage transition times. Parkinsonism Relat Disord. 2011;17(3):194-7.

60. Heinonen EH, Myllyla V. Safety of selegiline (deprenyl) in the treatment of Parkinson's disease. Drug Safety. 1998;19(1):11-22.

61. Karlsen K, Larsen JP, Tandberg E, Jørgensen K. Fatigue in patients with Parkinson's disease. Mov Disord. 1999;14(2):237-41.

62. Stocchi F, Abbruzzese G, Ceravolo R, Cortelli P, D'Amelio M, De Pandis MF, et al. Prevalence of fatigue in Parkinson disease and its clinical correlates. Neurology. 2014;83(3):215-20.

63. Finberg JP, Youdim MB. Pharmacological properties of the antiParkinson drug rasagiline; modification of endogenous brain amines, reserpine reversal, serotonergic and dopaminergic behaviours. Neuropharmacology. 2002;43(7):1110-8.

64. Parkinson Study Group. A controlled trial of rasagiline in early Parkinson disease: the TEMPO Study. Arch Neurol. 2002;59(12):1937-43. 
65. Rascol O, Brooks DJ, Melamed E, Oertel W, Poewe W, Stocchi $\mathrm{F}$, et al. Rasagiline as an adjunct to levodopa in patients with Parkinson's disease and motor fluctuations (LARGO, Lasting effect in Adjunct therapy with Rasagiline Given Once daily, study): a randomised, double-blind, parallel-group trial. Lancet. 2005;365(9463):947-54.

66. Parkinson Study Group. A controlled, randomized, delayed-start study of rasagiline in early Parkinson disease. Arch Neurol. 2004;61(4):561-6.

67. Hauser RA, Lew MF, Hurtig HI, Ondo WG, Wojcieszek J, FitzerAttas CJ. Long-term outcome of early versus delayed rasagiline treatment in early Parkinson's disease. Mov Disord. 2009;24(4):564-73.

68. Rascol O, Fitzer-Attas CJ, Hauser R, Jankovic J, Lang A, Langston JW, et al. A double-blind, delayed-start trial of rasagiline in Parkinson's disease (the ADAGIO study): prespecified and post-hoc analyses of the need for additional therapies, changes in UPDRS scores, and non-motor outcomes. Lancet Neurol. 2011;10(5):415-23.

69. Cattaneo C, Caccia C, Marzo A, Maj R, Fariello RG. Pressor response to intravenous tyramine in healthy subjects after safinamide, a novel neuroprotectant with selective, reversible monoamine oxidase B inhibition. Clin Neuropharmacol. 2003;26(4):213-7.

70. Chazot PL. Safinamide for the treatment of Parkinson's disease, epilepsy and restless legs syndrome. Curr Opin Investig Drugs. 2007;8(7):570-9.

71. Stocchi F, Borgohain R, Onofrj M, Schapira AH, Bhatt M, Lucini $\mathrm{V}$, et al. A randomized, double-blind, placebo-controlled trial of safinamide as add-on therapy in early Parkinson's disease patients. Mov Disord. 2012;27(1):106-12.

72. Barone P, Fernandez H, Ferreira J, Mueller T, Saint-Hilaire M, Stacy M, et al. Safinamide as an add-on therapy to a stable dose of a single dopamine agonist: results from a randomized, placebocontrolled, 24-week multicenter trial in early idiopathic Parkinson disease (PD) patients (MOTION Study). Neurology. 2013;80:Meeting abstracts 1 P01.061.

73. Schapira AH, Fox S, Hauser R, Jankovic J, Jost W, Kulisevsky J, et al. Safinamide add on to L-dopa: a randomized, placebo-controlled 24-week global trial in patients with Parkinson's disease and motor fluctuations (SETTLE). Neurology. 2013;80:Meeting Abstracts 1 P01.062.

74. Borgohain R, Szasz J, Stanzione P, Meshram C, Bhatt M, Chirilineau D, et al. Randomized trial of safinamide add-on to levodopa in Parkinson's disease with motor fluctuations. Mov Disord. 2014;29(2):229-37.

75. Gregoire L, Jourdain VA, Townsend M, Roach A, Di Paolo T. Safinamide reduces dyskinesias and prolongs L-DOPA antiparkinsonian effect in parkinsonian monkeys. Parkinsonism Relat Disord. 2013;19(5):508-14.

76. Sawada H, Oeda T, Kuno S, Nomoto M, Yamamoto K, Yamamoto M, et al. Amantadine for dyskinesias in Parkinson's disease: a randomized controlled trial. PloS One. 2010;5(12):e15298.
77. Borgohain R, Szasz J, Stanzione P, Meshram C, Bhatt MH, Chirilineau D, et al. Two-year, randomized, controlled study of safinamide as add-on to levodopa in mid to late Parkinson's disease. Mov Disord. 2014 Sep;29(10):1273-80.

78. Lipton SA, Rosenberg PA. Excitatory amino acids as a final common pathway for neurologic disorders. $\mathrm{N}$ Engl $\mathrm{J}$ Med. 1994;330(9):613-22.

79. Vaarmann A, Kovac S, Holmstrom KM, Gandhi S, Abramov AY. Dopamine protects neurons against glutamate-induced excitotoxicity. Cell Death Dis. 2013;4:e455.

80. Stocchi F, Arnold G, Onofrj M, Kwiecinski H, Szczudlik A, Thomas A, et al. Improvement of motor function in early Parkinson disease by safinamide. Neurology. 2004;63(4):746-8.

81. Stocchi F, Vacca L, Grassini P, De Pandis MF, Battaglia G, Cattaneo C, et al. Symptom relief in Parkinson disease by safinamide: Biochemical and clinical evidence of efficacy beyond MAO-B inhibition. Neurology. 2006;67(7 Suppl 2):S24-9.

82. Hauser RA, Silver D, Choudhry A, Eyal E, Isaacson S. Randomized, controlled trial of rasagiline as an add-on to dopamine agonists in Parkinson's disease. Mov Disord. 2014;29(8):1028-34.

83. Schapira AH, Stocchi F, Borgohain R, Onofrj M, Bhatt M, Lorenzana $\mathrm{P}$, et al. Long-term efficacy and safety of safinamide as add-on therapy in early Parkinson's disease. Eur J Neurol. 2013;20(2):271-80.

84. Marconi S, Zwingers T. Comparative efficacy of selegiline versus rasagiline in the treatment of early Parkinson's disease. Eur Rev Med Pharmacol Sci. 2014;18(13):1879-82.

85. Jost WH, Friede M, Schnitker J. Comparative efficacy of selegiline versus rasagiline in the treatment of early Parkinson's disease. Eur Rev Med Pharmacol Sci. 2014;18(22):3349.

86. Jost WH, Friede M, Schnitker J. Indirect meta-analysis of randomised placebo-controlled clinical trials on rasagiline and selegiline in the symptomatic treatment of Parkinson's disease. Basal Ganglia. 2012;2(4, Supplement):S17-26.

87. Nutt JG, Bloem BR, Giladi N, Hallett M, Horak FB, Nieuwboer A. Freezing of gait: moving forward on a mysterious clinical phenomenon. Lancet Neurol. 2011;10(8):734-44.

88. Schapira AH. Rasagiline in neurodegeneration. Exp Neurol. 2008;212(2):255-7.

89. Schapira AH, Obeso J. Timing of treatment initiation in Parkinson's disease: a need for reappraisal? Ann Neurol. 2006;59(3):559-62.

90. Marsden CD. Parkinson's disease. Lancet. 1990;335(8695): 948-52.

91. Knudsen Gerber DS. Selegiline and rasagiline: twins or distant cousins? Consult Pharm. 2011;26(1):48-51.

92. Miklya I. Essential difference between the pharmacological spectrum of (-)-deprenyl and rasagiline. Pharmacol Rep. 2014;66(3):453-8.

93. Robottom BJ. Efficacy, safety, and patient preference of monoamine oxidase B inhibitors in the treatment of Parkinson's disease. Patient Prefer Adher. 2011;5:57-64. 